\title{
Inhibition of cGMP-metabolizing PDEs as target for cognitive enhancement
}

\author{
Holger Rosenbrock \\ From 7th International Conference on cGMP Generators, Effectors and Therapeutic Implications \\ Trier, Germany. 19-21 June 2015
}

\begin{abstract}
Background
Inhibition of specific phosphodiesterases (PDEs) in the brain has gained attention as a potential new approach for memory enhancement. Among those PDEs are PDE2A and PDE9A which are either, in case of PDE2A, dualspecific for cyclic guanosine and cyclic adenosine monophosphate (cGMP and cAMP, respectively) or selective for cGMP, in case of PDE9A. Both PDEs are expressed in cognition relevant regions of the brain, and in these regions, especially cGMP plays an important role as second messenger in neurons related to NMDA receptor signalling facilitating synaptic plasticity and memory formation. Thus, PDE2A and PDE9A inhibitors are hypothesized to improve cognitive function via improving glutamatergic neurotransmission and/or signalling pathways leading to strengthening of synaptic plasticity. In order to characterize mechanistically the effects of PDE2A and PDE9A, selective inhibitors of both PDEs were tested on long-term potentiation (LTP) in rat hippocampal slices, a widely accepted cellular experimental model of synaptic plasticity and memory formation as well as on hippocampal pairedpulse facilitation (PPF), a model to assess presynaptic function. Furthermore, both inhibitors were evaluated regarding enhancement of cyclic nucleotide levels in brain and memory performance in rodent cognition tasks.
\end{abstract}

\section{Results}

Both, the PDE2A inhibitor PF-999 [1] and the PDE9A inhibitor Bay 73-6691 [2] increased hippocampal LTP, but in contrast to PDE2A inhibition, PDE9A inhibition not only enhances early and late LTP, but even transforms early LTP into late LTP. Regarding effects on hippocampal PPF, only the PDE2A inhibitor but not the PDE9A inhibitor was able to modulate presynaptic function, indicating that

Correspondence: holger.rosenbrock@boehringer-ingelheim.com Department of CNS Diseases Research, Boehringer Ingelheim Pharma GmbH \& Co KG, Bibearch, Germany 